\title{
Catalytic Methanolysis Induced by Succinoglycan, a Rhizobial Exopolysaccharide
}

\author{
Simook Kang, ${ }^{\dagger}$ Sanghoo Lee, ${ }^{\ddagger}$ Sanggyu Kyung, ${ }^{\dagger}$ and Seunho Jung ${ }^{\dagger, \$, \S, *}$ \\ ${ }^{\dagger}$ Department of Advanced Technology Fusion, Konkuk University, Seoul 143-701, Korea. *E-mail: shjung@konkuk.ac.kr \\ \$Bio/Molecular Informatics Center, Konkuk University, Seoul 143-701, Korea \\ $\S^{\S}$ Department of Bioscience and Biotechnology \& Department of Microbial Engineering, College of Engineering, \\ Konkuk University, Seoul 143-701, Korea \\ Received March 20, 2006
}

Key Words : Succinoglycan, Rhizobium meliloti, Catalytic carbohydrate, Methanolysis, 7-Acetoxy-4-methylcoumarin

Nitrogen-fixing soil bacterium, $R$. meliloti, closely associates with a specific host plant through the induction and invasion of root hair nodules. ${ }^{1,2}$ During the process, the bacterial polysaccharides play crucial roles for the successful nitrogen fixation. $R$. meliloti has been reported to produce two EPSs, succinoglycan (acidic extracellular polysaccharide) and EPS II, for the effective invasion of alfalfa by $R$. meliloti. The symbiotically active forms of these polysaccharides may function as signal molecules to facilitate the nodule invasion process. ${ }^{3,4}$ The succinoglycan is a biopolymer of octasaccharide repeating units. The biopolymer consists of one galactose and seven glucose units and has approximately one acetyl, one succinyl, and one pyruvyl modification per a repeating subunit (Fig. 1). Wild-type $R$. meliloti produces succinoglycan in lowmolecular weight (LMW) forms, consisting of short oligomers of the octasaccharide repeating unit, and in highmolecular weight (HMW) forms, consisting of hundreds to thousands of repeating units., ${ }^{3,5}$

Protein enzymes, ribozymes, ${ }^{6}$ DNAzymes, ${ }^{7}$ and catalytic antibodies $^{8}$ as the typical biocatalysts have been studied by many researchers in various fields, except for catalytic carbohydrates derived from living organisms. Cyclodextrins (cyclic $\alpha-(1,4)$-glucans) as a non-natural carbohydrate have

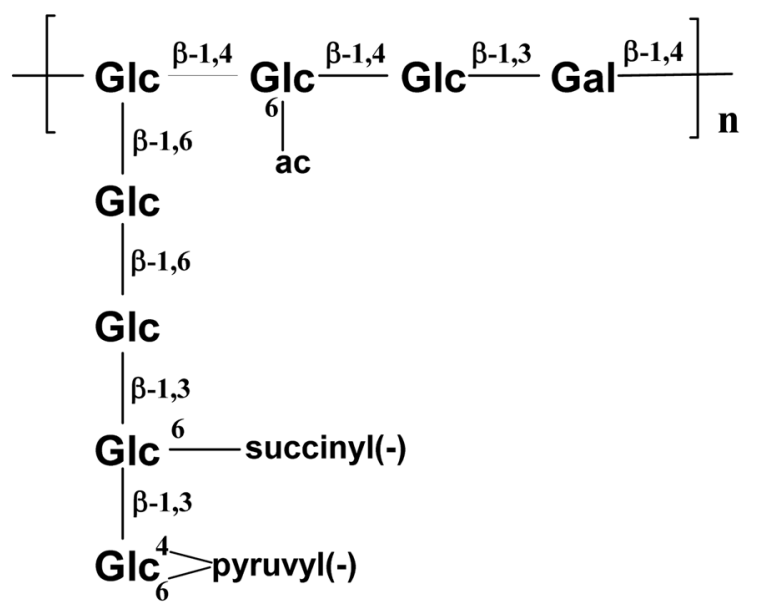

Figure 1. The repeating unit of the succinoglycan isolated from $R$. mellioti $1021 .^{15}$ been reported to show chiral recognitions, ${ }^{9}$ inclusion complexations ${ }^{10}$ and catalytic functions. ${ }^{11}$ However, the novel function of cyclic $\beta$-1,2-D-glucan (cyclosophoraose), as a catalyst for methanolysis reaction, produced by $R$. meliloti 2011 has been recently reported by the previous study. ${ }^{12}$ According to the previous work, the cyclooligosaccharide enhanced dramatically the rates of the methanolysis reactions of both oxazolone compounds and phospholipids via acyl intermediates. This result made it possible to advance further studies on catalytic functions of other microbial complex carbohydrates.

Coumarin and its derivatives, flavonoid isolated from various plants, have been generally reported as antioxidants. ${ }^{13}$ Here, we report, for the first time, that a microbial acidic polysaccharide, succinoglycan, functions as a novel catalyst for methanolysis of 7-acetoxy-4-methylcoumarin, one of coumarin derivatives. The conversion rate was then quantitatively investigated through ${ }^{1} \mathrm{H}$ NMR spectroscopic analysis.

The structure of the isolated succinoglycan was identified through FT-IR and ${ }^{1} \mathrm{H}$ NMR spectroscopic analyses. The FTIR spectrum of the succinoglycan produced by $R$. meliloti 1021 exhibits some characteristic peaks. The peak at around $3,425 \mathrm{~cm}^{-1}$ indicates the $\mathrm{O}-\mathrm{H}$ stretching frequency of the sugar poly hydroxyl groups and the absorption at $2,923 \mathrm{~cm}^{-1}$ is attributed to $\mathrm{C}-\mathrm{H}$ stretching in the sugar backbone. Absorption peak at $1,618 \mathrm{~cm}^{-1}$ is assigned to the symmetric stretching of the carboxyl groups and the absorption at 1,400 $\mathrm{cm}^{-1}$ is possibly due to the bending tendency of symmetric $\mathrm{CH}_{3}$ groups within the acetyl and pyruvyl groups as a substituent and the absorption at $1,072 \mathrm{~cm}^{-1}$ is attributed to an asymmetric $\mathrm{C}-\mathrm{O}-\mathrm{C}$ stretching band resulting from the sugar backbone (Figure 2(A)). These absorption patterns are similar to those that found in typical microbial acidic EPS as reported previously. ${ }^{16}{ }^{1} \mathrm{H}$ NMR spectrum of the succinoglycan is also shown in Figure 2(B). The chemical shifts at 1.51 and 2.19 ppm represent the methyl protons of the 1carboxyethylidene (pyruvate) and acetyl groups, respectively. The signals of the intense resonance at 2.58 and 2.69 ppm represent the methylene protons of the succinyl group. The complex region from 3.30 to $4.90 \mathrm{ppm}$ represents the protons of the sugar backbone constituents. The solvent peak 

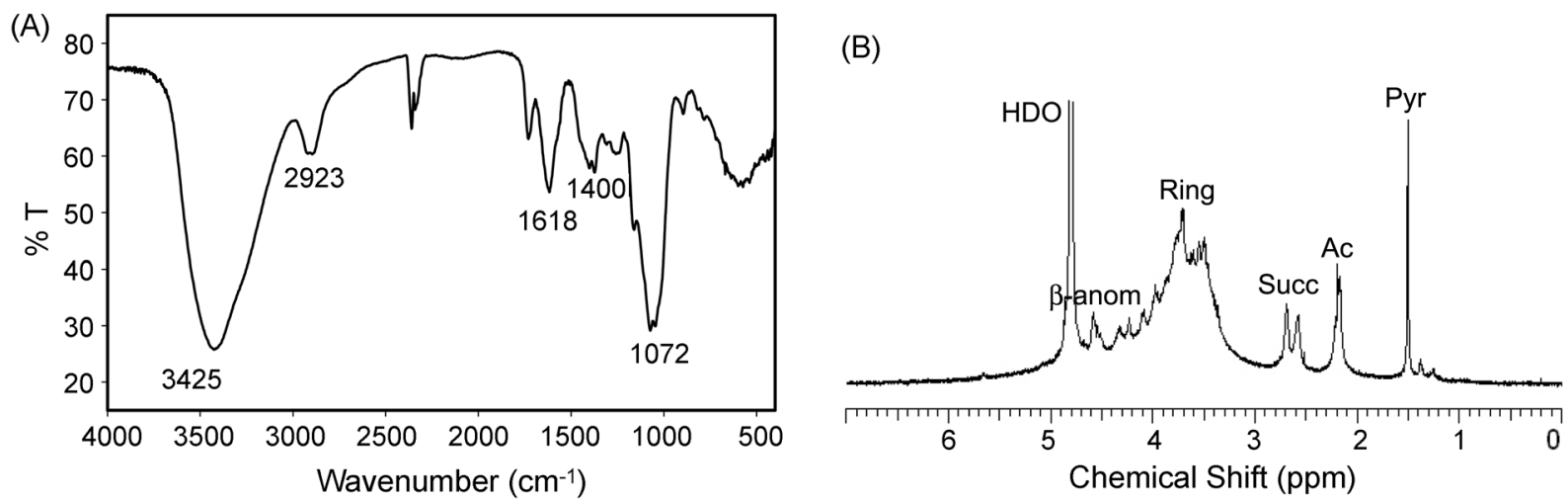

Figure 2. Structural identification of succinoglycan isolated from $R$. meliloti 1021. (A) The FT-IR spectrum of succinoglycan. (B) ${ }^{1} \mathrm{H}$ NMR spectrum of succinoglycan. $\beta$-anom, $\beta$-anomeric protons; ring, ring protons of the sugars; succ, methyl protons of the succinyl groups; ac, methyl protons of the acetyl groups; pyr, methyl protons of the 1-carboxyethylidene (pyruvate) groups.

(A)

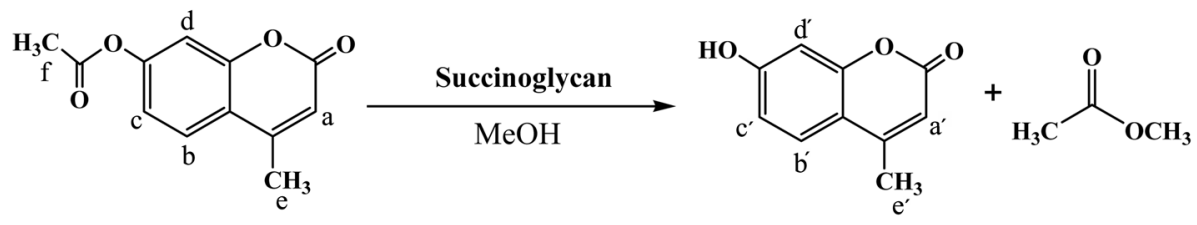

(B)

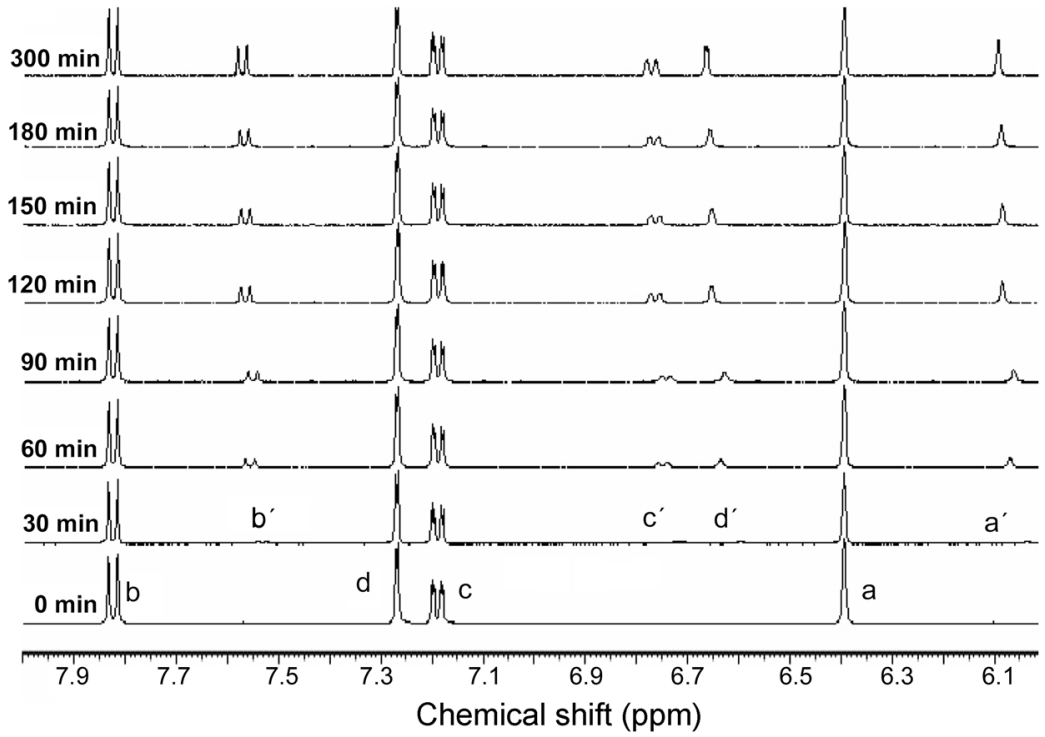

(C)

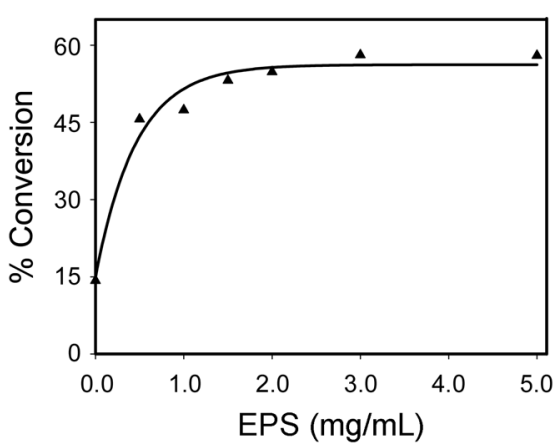

(D)

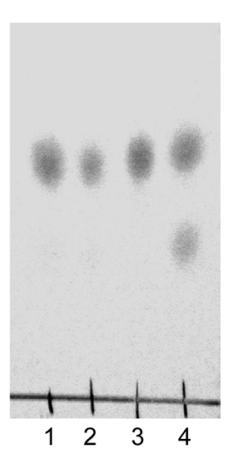

Figure 3. Methanolysis reaction of 7-acetoxy-4-methylcoumarin catalyzed by succinoglycan. (A) Scheme for methanolysis of 7-acetoxy-4methylcoumarin by succinoglycan. (B) Partial ${ }^{1} \mathrm{H}$ NMR spectra of 7-acetoxy-4-methylcoumarin in the presence of succinoglycan as catalyst at $0,30,60,90,120,150,180$ and $300 \mathrm{~min}$. (C) Conversion of 7-acetoxy-4-methylcoumarin with increasing amount of succinoglycan added. (D) TLC image of the reaction mixtures of 7-acetoxy-4-methylcoumarin and some carbohydrates containing succinoglycan after 300 $\min$ at $30^{\circ} \mathrm{C}$. Lane 1,7-acetoxy-4-methylcoumarin alone; Lane 2, glucose; Lane 3, $\beta$-CD; Lane 4, succinoglycan. 
(HOD) was observed at $4.80 \mathrm{ppm} .{ }^{1} \mathrm{H}$ NMR spectroscopic analysis indicated that the HMW fractions consisted of fully acylated succinoglycan containing approximately one succinyl, one acetyl, and one pyruvate modification per oligosaccharide subunit. Study on structural analyses of succinoglycan produced by $R$. melilioti 1021 was also reported..$^{5}$

Microbial succinoglycan catalyzed the ester cleavage of 7acetoxy-4-methylcoumarin under methanol solution as shown in Figure 3(A). Figure 3(B) shows the partial ${ }^{1} \mathrm{H}$ NMR spectra indicating the progresses of the catalytic reaction at different periods of time. The ring protons of the cleaved coumarin derivative clearly appeared at $30 \mathrm{~min}$ in the presence of the Rhizobial succinoglycan. Based on the ${ }^{1} \mathrm{H}$ NMR spectra measured, time-course curves were obtained where the reaction was enhanced about 6 -fold $\left(k_{\text {cat }} /\right.$ $\left.k_{\text {uncat }}\right)$ in the presence of $5 \mathrm{mg}$ of succinoglycan in $\mathrm{MeOH}$ (1 $\mathrm{mL}$ ) at $30{ }^{\circ} \mathrm{C}$. Figure $3(\mathrm{C})$ shows the dependence of the cleavage reaction as added amount of succinoglycan. After $300 \mathrm{~min}$ at $30{ }^{\circ} \mathrm{C}$, the conversion was saturated when amount of succinoglycan added was $3 \mathrm{mg}$. Through TLC analysis, we also investigated the methanolysis reaction to identify the effect of other typical carbohydrates, where glucose and $\beta$-cyclodextrin (CD) were used. After reaction for $300 \mathrm{~min}$ at $30{ }^{\circ} \mathrm{C}$, a phosphoimage of the reaction mixtures on UV radiator is shown in Figure 3D. As a product, the ring moiety of 7-acetoxy-4-methylcoumarin cleaved by succinoglycan was observed on TLC. On the contrary, catalytic effects were hardly detected when the other typical carbohydrates of the same amount to succinoglycan were added.

Although the same experiments were also performed with 4-phenyl-1(2H)-pathalazinone and 2,3,4,5-tetrahydro-6phenyl-3(2H)-pyridazinone as the compounds containing amide group, no methanolysis reactions were observed by even adding succinoglycan. The results indicate that the acidic polysaccharide as a catalyst has more favorable accessibilities for carbonyl groups within common ester compounds than for carbonyl groups within amide bonds. The catalytic methanolysis of the coumarine derivative could be primarily attributed to the hydroxyl or carboxyl groups of the microbial acidic polysaccharide, like as the oxazolone compound catalyzed by Rhizobial cyclic $\beta-(1,2)-$ glucan. ${ }^{12}$

Combined with all the data obtained, it is assumed that a hypothetical mechanism of the catalytic reaction might be similarly proposed as our report previously. ${ }^{12}$ The molecule could be first activated by the succinoglycan through hydrogen bonding. Then, the reaction could progress further by an attack of hydroxyl groups of $\beta$-gluco- or galactopyranose, and/or succinyl or pyruvyl groups as substituent components of the polysaccharide via acyl intermediates. Considering an intermediate formed between the oxazolone compound and cyclic $\beta$-(1,2)-glucan derived from $R$. meliloti, it may be reasonable that the reaction catalyzed by the succinoglycan would also occur via acyl intermediates, though an acyl intermediate formed during the methanolysis of the coumarin compound catalyzed by succinoglycan could not detect through both NMR and mass spectrometric analysis because of very high molecular weight of the acidic polysaccharide.

In this study, microbial succinoglycan involved in symbiosis between Rhizobium species and its specific host plants, functioned as a catalytic carbohydrate in the methanolysis reaction of 7-acetoxy-4-methylcoumarin, a derivative of flavonoid, though the acidic polysaccharide itself plays important roles in initiation and elongation of infection threads during successful nodulation process. ${ }^{17}$ The acidic succinoglycan enhanced the ester cleavage reaction about 6fold for the coumarin at $30{ }^{\circ} \mathrm{C}$ in methanol. The result suggests that the microbial acidic succinoglycan have potentials as a novel catalytic carbohydrate for methanolysis reactions of various ester compounds under methanol environment. Although the exact reaction mechanism remains unclear, we assume that the catalytic methanolysis reaction could be attributed to not only an attack of hydroxyl or the acidic substituent groups within the microbial succinoglycan as a strong nucleophile against the electrophilic sites of the coumarin derivative compound but also a specific three dimensional structure of the complex carbohydrate via acyl intermediates in methanol environment. Exact mechanistic study of the methanolysis catalyzed by zooglan is in progress.

\section{Experimental Section}

7-Acetoxy-4-methylcoumarin was purchased from Aldrich Chemical Co. (Milwaukee,WI, USA), and 4-phenyl-1(2H)phthalazionoe and 2,3,4,5-tetrahydro-6-phenyl-3(2H)-pyridazinone from Indofine Chemical Co. (Hillsborough, NJ, USA). Methanol of HPLC grade (99.9\%) was used as a reaction solvent. DMSO- $d_{6}$ as NMR solvent was purchased from Sigma Chemical Co. (St. Louis, MO, USA).

$R$. meliloti 1021 was cultivated in a glutamate-mannitolsalts (GMS) medium ( $\mathrm{pH}$ 7.0) supplemented with biotin, thiamine, and trace elements as described by previous report. ${ }^{11}$ Typically, $500 \mathrm{~mL}$ of the GMS medium in a 1-liter Erlenmeyer flask was inoculated with $25 \mathrm{~mL}$ of $R$. meliloti 1021 overnight culture and cultivated at $30^{\circ} \mathrm{C}$ for 7 days with shaking $(250 \mathrm{rpm})$. Cells were centrifuged at 20,000 g for $30 \mathrm{~min}$, and the supernatant that contains the secreted EPS was concentrated in vacuum by evaporation. To separate high-molecular weight (HMW) succinoglycan, the concentrated material was suspended in $100 \mathrm{~mL}$ of $0.1 \mathrm{M} \mathrm{NaCl}$, to which 3 volumes of ethanol were added with stirring. The HMW succinoglycan turned into a gelatinous precipitate and was then collected by centrifugation at $6,000 \mathrm{~g}$ for $30 \mathrm{~min}$. Alcohol precipitation step was repeated 2 times, and then the HMW succinoglycan was dialyzed (MWCO > 12,400) against distilled water and then lyophilized. ${ }^{5,15}$

A pellet for Fourier transform-infrared (FT-IR) analysis was obtained by carefully grinding $2 \mathrm{mg}$ of succinoglycan with a $\mathrm{KBr}$ and then pressing in a mold. The FT-IR spectrum of succinoglycan was obtained using a JASCO FT-IR-300E spectrometer (REV, USA) over the wavenumber of 4000$400 \mathrm{~cm}^{-1}$. Samples for proton nuclear magnetic resonance 
$\left({ }^{1} \mathrm{H}\right.$ NMR) spectroscopic analysis were sonicated, lyophilized, and then dissolved at $10 \mathrm{mg} / \mathrm{mL}$.

7-Acetoxy-4-methylcoumarin was first dissolved in methanol $(1 \mathrm{~mL})$ and then followed by adding succinoglycan. The reaction mixture was stirred in a capped round flask with the volume of $5 \mathrm{~mL}$ and an aliquot was taken for thinlayer chromatography (TLC) and ${ }^{1} \mathrm{H}$ NMR analysis.

To monitor the reaction, the mixture was assayed by TLC. Silica Gel 60 F254 glass-backed TLC plates were spotted with the analytes and developed with solvent systems ( $3: 1$ $=$ hexane:ethyl acetate). The reactant and product were identified by irradiation with ultraviolet light $(254 \mathrm{~nm}) .^{12}$

NMR spectroscopic analysis was carried out on a Bruker Avance 500 spectrometry. Conversion of 7-acetoxy-4methylcoumarin was determined by measuring the decrease of the determined integral area of the resonance at $7.82 \mathrm{ppm}$ [proton (d) of reactant] and increase of the integral area of the resonance at $7.59 \mathrm{ppm}$ [proton (d') of product] by ${ }^{1} \mathrm{H}$ NMR spectroscopy (Figure 3(B)). The reacted fractions were then quantified. For this reaction, the whole reactant was taken at a given time, rapidly evaporated under reduced pressure, and then placed under vacuum to remove the residual methanol before NMR measurement. The measurements were done in DMSO- $d_{6}$, and then tetramethylsilane (TMS) was used as an internal standard. The time course of the methanolysis reaction was fitted to a single exponential to obtain kinetic parameters. ${ }^{12}$

Acknowledgements. This research was supported by Konkuk University in 2005.

\section{References}

1. Finan, T. M.; Hirsch, A. M.; Leigh, J. A.; Johansen, E.; Kuldau, G. A.; Deegan, S.; Walker, G. C.; Signer, E. R. Cell 1985, 40, 869.

2. Leigh, J. A.; Signer, E. R.; Walker, G. C. Proc. Nat. Acad. Sci. USA 1985, 82, 6231.

3. Battisti, L.; Lara, J. C.; Leigh, J. A. Proc. Nat. Acad. Sci. USA 1992, 89, 5625.

4. Gonza'lez, J. E.; Reuhs, B. L.; Walker, G. C. Proc. Nat. Acad. Sci. USA 1996, 93, 8636.

5. Leigh, J. A.; Lee, C. C. J. Bacteriol. 1988, 170, 3327.

6. Cech, T. R.; Zaug, A. J.; Grabowski, P. J. Cell 1981, 27, 487.

7. Breaker, R. R.; Joyce, G. F. Chem. Biol. 1994, 1, 223.

8. Ulrich, H. D.; Mundorff, E.; Santarsiero, B. D.; Driggers, E. M.; Stevens, R. C.; Schultz, P. G. Nature 1997, 389, 271.

9. Choi, S. H.; Noh, H. J.; Lee, K. P. Bull. Korean Chem. Soc. 2005 26(10), 1549 .

10. Choi, Y. J.; Lee, J. H.; Cho, K. W.; Hwang, S. T.; Jeong, K. J.; Jung, S. H. Bull. Korean Chem. Soc. 2005, 26, 1203.

11. Eastburn, S. D.; Tao, B. Y. Biotechnol Adv. 1994, 12(2), 325.

12. Lee, S. H.; Jung, S. H. Carbohydr. Res. 2004, 339, 461.

13. Konstantina, C. F.; Dimitra, J. H.; Konstantinos, E. L.; Demetrios, N. N. Current Pharmaceutical Design 2004, 10, 3813.

14. Zevenhuizen, L. P. T. M.; Van Neerven, A. R. W. Carbohydr. Res. 1983, 118,127

15. Wang, L. X.; Wang, Y.; Pellock, B.; Walker, G. C. J. Bacteriol. 1999, 181, 6788.

16. Friedman, B. A.; Dugan, P. R.; Pfister, R. M.; Remsen, C. C. J. Bacterol. 1968, 96, 2144.

17. Cheng, H. P.; Walker, G. C. J. Bacteriol. 1998, 180, 5183.

18. Reinhold, B. B.; Chan, S. Y.; Reuber, L.; Marra, A.; Walker, G. C.; Reinhold, V. N. J. Bacteriol. 1994, 176, 1997. 